\title{
Is Precision Medicine Consistent With Primary Care?
}

Dana E. King, MD, MS

(Fam Med. 2019;51(3):224-6.)

doi: 10.22454/FamMed.2019.687886

C lose your eyes. Imagine it is 10 years from now, and you are in your medical office. You are in a small televideo cubicle preparing for a well visit with one of your patients...in the next town. After morning telerounds from 7:30am-10:00am, you move out into your exam area. Your medical assistant has already roomed the patient, completed the initial history, pended the orders for the flu and pneumonia vaccines, and warmed up the handheld ultrasound machine. You greet the familiar patient, review the history, and conduct the exam, which you do with the aid of your electronic stethoscope. You decide that the patient needs to start taking blood pressure medication. You explain the need for treatment, reviewing the guidelines and how they individually apply to him. A robot arm extends from beside the computer terminal and pricks the patient's finger to obtain the blood specimen. You tell the patient you will be back in a few minutes with the results from his chemistry and DNA analysis, which will inform your decision to start an ACE inhibitor, an ARB, or a calcium channel blocker. This is precision medicine in primary care.

Precision medicine is the provision of medical care using precise genetic information to predict response to treatment and to customize care for a specific individual. The National Institutes of Health $(\mathrm{NIH})$ web page on genetics states it this way: "an emerging approach for disease treatment and prevention that takes into account individual variability in genes, environment, and lifestyle for each person."

Pharmacogenomics is a subset of the precision medicine field applied specifically to predict an individual's response to drugs. At present, pharmacogenomics is in its relative infancy and is used only in a limited number of situations, many of which are outside the primary care arena. However, pharmacogenomics applications and other precision medicine techniques are expanding rapidly and will increasingly become integrated into patient care. ${ }^{2}$ Targeted pharmacogenomic-based cancer therapy is another area in which advancements are being accomplished on a regular basis. Targeted therapy directs its activity on a specific molecule or group of molecules, rather than using a less-focused approach of killing rapidly dividing cells. ${ }^{3}$

Is precision medicine consistent with primary care? The field is advancing quickly, perhaps beyond helping to make medication choices easier. One argument against integration is based on the presupposition that precision medicine might evolve and replace primary care and make family physicians superfluous to the process of patient care. Perhaps the emphasis on molecular medicine, genetic profiles, and an impersonal practice of medicine based on laboratory tests goes too far. A clinical practice based on precision medicine might reduce human beings to little more than data, as some critics say, and sometimes data can be wrong. ${ }^{4}$

The debate regarding whether precision medicine should be integrated into clinical care is ongoing. Diane Korngiebel, DPhil, University of Washington, is one of several people who are exploring the ethical challenges of precision medicine. ${ }^{5}$ The very nature of being able

From the Department of Family Medicine, West Virginia University, Morgantown, WV. 
to know the future (genetic markers of cancer in your future) and its challenge to our humanness, is at the heart of many people's reluctance to embrace this new technology. When a patient can get his or her own genetic profile online, complete with diagnoses and carrier states, will primary care physicians be necessary? Empowering patients has been a goal of primary care for decades, but data without guidance from physicians could be hazardous. It is not unrealistic to foresee a future in which primary care could be replaced by a new specialty of epigenetic technicians who are a combination of physician assistants and computer programmers.

Even among advocates, the nature of epigenetics and precision medicine also present a number of barriers to adoption into primary care. The first is the vast complexity and the need for physicians to learn about a huge array of new tests and learn to counsel patients using probability-based decision making. The challenges to the continuing medical education industry are enormous, for specialists and primary care physicians alike. Learning the application of the human genome to common conditions may require physicians to do extra continuing education to become experts.

Data sharing platforms and security for precision medicine data are another problem. Will servers and data files be private? How do physicians access the data in a secure way that protects patients' privacy? The NIH has a working policy on privacy and trust principles to guide precision medicine. ${ }^{6}$ The NIH document articulates an excellent set of core values for security and access to data, but a policy by itself cannot assure the public of the security needed to protect privacy and lack of manipulation of data by employers, insurers, or private citizens.

Costs for precision medicine likely will also be an issue. Current genetic testing is specialty oriented, and consultation for applying the results adds expense. At present, costs for various aspects of precision medicine are not fully known, but an entire genome test can cost $\$ 3,000-\$ 5,000$ at some labs. However, one lab recently offered a lower price of $\$ 699$, so costs may be coming down (dantelabs.com). However, reconciling one's genome results and comparing it to known abnormal entities in a clinically meaningful way will likely again raise costs into the $\$ 1,000$ s.

Despite these challenges, precision medicine use is expanding. It is currently used most often in specialized medical conditions such as in cancer, hematology, and specific genetic diseases. For example, the use of combined immunotherapy and specific molecule-targeted therapies has brought promise and hope in previously difficult-to-treat cancers such as small cell lung cancer. ${ }^{7}$ Primary care physicians may some day be able to use moleculetargeted therapies in earlier stages of lung cancer and a variety of other conditions as the field develops and expands.

Precision medicine is not limited to drugs or cancer. New targets are being explored and developed in many other areas, including diabetic kidney disease, autoimmune liver disease, psychiatry, and many others that overlap with primary care ${ }^{8-10}$ Researchers are exploring the use of precision medicine for common chronic diseases treated daily by primary care physicians. For example, physicians routinely screen for albuminuria, but they have limited options in management of patients with diabetes and early signs of kidney damage. New precision targeted therapies will expand options for such patients. ${ }^{8}$

Warfarin therapy, notorious in primary care offices for the difficulty in managing an individual's response, is now a new frontier in precision medicine. New algorithms and testing cascades are being developed that will soon make it to routine primary care practice. While only $35 \%$ of the variation in dosing response in warfarin is due to genetics, new approaches that account for demographics and diet as well as genetics are being perfected so improved warfarin management techniques can be used more widely in primary care. ${ }^{11}$

Primary care physicians are well positioned to use the precision medicine tools of the future to enhance patient management. Precision medicine is in the process of identifying many relevant DNA and RNA regions that predict response to a variety of medications, including those used to treat blood pressure, cancer, pain, asthma, and more. ${ }^{12-14}$ The tools need further development and the ability to access results in a timely manner. When that occurs, the new era will arrive.

So, let us reexamine the original question: Is precision medicine consistent with primary care? I believe that primary care practices likely will evolve and use these newly developed precision medicine techniques as careenhancing tools, similarly to the way they use common laboratory tests and CT scans now-as an adjunct to history and physical diagnosis in the management of the patient. Genetic profiles alone cannot take into account 
the demographics, social situations, lifestyle habits, and patient perspectives, but a primary care physician can do so. The crucial social, environmental, and lifestyle factors that contribute to risk and chronic disease mitigation will need to be assimilated and managed by a primary care physician, in the context of a longitudinal doctor-patient relationship. Family physicians are trained to take into account all aspects of the patient's profile to address their medical and psychosocial well-being.

In the future scenario described at the start of this paper, the physician was seeing a longtime patient and providing comprehensive, longitudinal care. The doctor assessed the patient's lifestyle, environment, and genetic factors, and explained the diagnosis, prognosis, and need for treatment. DNA-typing and SNP analysis of the relevant portion of the human genome was available immediately or within 24 hours. The prescription for blood pressure medication in the example case-a decision that is currently based on guidelines from large evidence-based studies and guidelines of expert panels—will in the future be based on the individual's exact human gene profile and personal characteristics. Future family physicians will still be doing compassionate, comprehensive, continuity-based primary care, but will be operating in a new world of precision medicine that is highly incorporated into primary care. Precision medicine is not only consistent with primary care, some day it will be central to providing it.

You can open your eyes now.

CORRESPONDENCE: Address correspondence to Dr Dana King, West Virginia University School of MedicineFamily Medicine, Box 9152, Robert C. Byrd Health Sciences Center, Morgantown, WV 26506. 304-598-6920. kingdana@wvuhealthcare.com.

\section{References}

1. National Library of Medicine. What is precision medicine? In: Genetics Home Reference; 2018. https://ghr.nlm.nih.gov/ primer/precisionmedicine/definition. Accessed October 19, 2018.

2. Fountzilas E, Tsimberidou AM. Overview of precision oncology trials: challenges and opportunities. Expert Rev Clin Pharmacol. 2018;11(8):797-804

3. Kantarjian HM, Keating MJ, Freireich EJ. Toward the potential cure of leukemias in the next decade. Cancer 2018;124(22):4301-4313.
4. Graber, Cythia. The problem with precision medicine. The New Yorker. Feb 5, 2015. https://www.newyorker.com/tech/ annals-of-technology/problem-precision-medicine. Accessed November 28, 2016.

5. Korngiebel DM, Thummel KE, Burke W. Implementing precision medicine: the ethical challenges. Trends Pharmacol Sci. 2017;38(1):8-14.

6. National Institutes of Health All of Us Research Program. Precision Medicine Initiative: Data Security Policy Principles and Framework Overview. https://allofus.nih.gov/ about/program-overview/precision-medicine-initiative-datasecurity-policy-principles-and-framework-overview. Accessed January 22, 2019

7. Liang H, Liu X, Wang M. Immunotherapy combined with epidermal growth factor receptor-tyrosine kinase inhibitors in non-small-cell lung cancer treatment. OncoTargets Ther. 2018;11:6189-6196.

8. Leti F, Morrison E, DiStefano JK. Long noncoding RNAs in the pathogenesis of diabetic kidney disease: implications for novel therapeutic strategies. Per Med. 2017;14(3):271-278.

9. Bossen L, Gerussi A, Lygoura V, Mells GF, Carbone M, Invernizzi P. Support of precision medicine through riskstratification in autoimmune liver diseases - histology, scoring systems, and non-invasive markers. Autoimmun Rev. 2018;17(9):854-865.

10. US Department of Veterans Affairs Health Services Research and Development. VA Research Funds New Initiative to Implement Genomic Test Results to Improve Depression Care for Veterans. https://www.hsrd.research.va.gov/news/ research_news/genomics-100316.cfm. Published October 3, 2016. Accessed January 22, 2019.

11. Bartlett G, Dawes M, Nguyen Q, Phillips MS. Precision Medicine in Primary Health Care. In: Varma M, Barh D, eds. Progress and challenges in precision medicine. Amsterdam: Elsevier/Academic Press; 2017:101-113.

12. Kelley EF, Snyder EM, Alkhatib NS, et al. Economic evaluation of a pharmacogenomic multi-gene panel test to optimize anti-hypertension therapy: simulation study. J Med Econ. 2018;21(12):1246-1253.

13. Webster LR, Belfer I. Pharmacogenetics and Personalized Medicine in Pain Management. Clin Lab Med. 2016;36(3):493-506.

14. Canonica GW, Ferrando M, Baiardini I, et al. Asthma: personalized and precision medicine. Curr Opin Allergy Clin Immunol. 2018;18(1):51-58. 